\title{
A general description of the subtidal habitats of the Dampier Archipelago, Western Australia
}

\author{
Peter F. Morrison \\ Sinclair Knight Merz Pty. Ltd., $7^{\text {th }}$ Floor Durack Centre, PO Box H615 \\ 263 Adelaide Terrace, Perth, Western Australia 6001, Australia \\ email: PMorrison@skm.com.au
}

\begin{abstract}
Subtidal habitats at 45 locations were recorded on video during the diving surveys of the Dampier Archipelago in 1998 (DA1/98) and 1999 (DA3/ 99) and analysed for percentage cover in terms of 13 benthic categories. The video recording provided a permanent record of each station while the video transect analysis provided a quantitative description of the benthic habitat to complement the semi-quantitative specimen collections. A statistical comparison of the habitats present indicated that the survey stations could be grouped into seven distinct categories (abiotic - six stations; macroalgae four stations; gorgonian soft corals - one station; neptheid and alcyonid soft corals - five stations; foliose non-Acropora hard corals - one station; branching Acropora - two stations; massive non-Acropora - four stations) and one less distinct category (abiotic and hard coral habitats, predominantly massive nonAcropora or tabulate Acropora species - twenty two stations) based on dominant percentage cover.
\end{abstract}

\section{INTRODUCTION}

The aim of this work was to provide a permanent record of 45 subtidal habitats in the Dampier Archipelago and to describe benthic composition at a functional group level. Biological sampling for the survey was conducted along a $25.0 \mathrm{~m}$ transect at each station. Of necessity, however, the band width of the transect varied for each taxonomic group investigated. Biodiversity and benthic assemblage composition varies with habitat type and for comparative purposes stations have been categorised.

\section{MATERIALS AND METHODS}

Video recordings at each of 45 stations, 19 in 1998 (DA1/98) and 26 in triplicate (78 transects) in 1999 (DA3/99), were undertaken. Recordings were acquired by movement of a Sony CCD Handycam Video 8 camera in a housing along $25.0 \mathrm{~m}$ long transects, according to the protocol developed by the Australian Institute of Marine Science (Carleton and Done, 1995). Each transect was marked by laying a $25.0 \mathrm{~m}$ measuring tape along the sea bed at relatively uniform depths. A SCUBA diver maintained a constant speed of $0.2 \mathrm{~m} / \mathrm{sec}$. and the video was kept $0.5 \mathrm{~m}$ above the surface of the habitat. This captured a band width of approximately 0.6 metre and a total area of $15.0 \mathrm{~m}^{2} /$ transect. Data on the depth and habitat type at each station are provided in Tables 1 and 2 while the locations of the stations are illustrated in Figure 1.
Video recordings of the 45 stations were captured electronically, saved to file and then stored on compact discs. The Sinclair Knight Merz Video Transect Analysis System then retrieved the electronically-recorded transect for analysis. The program randomly selects 200 frames from approximately 3,125 recorded along the 25.0 metre transect. Each frame was allocated one randomly placed spot. The substratum types beneath the respective spots were assigned a benthic descriptor.

Once a benthic descriptor was assigned and the respective frame completed, the program advanced the transect to the next randomly-selected frame and this process was repeated until the designated number of frames were completed. Upon completion, the program computed tallied counts and percentage cover (Osborne and Oxley, 1997). The data for each station were exported into an Excel spreadsheet for graphical presentation.

Since the purpose of the transect analysis was to provide a general description of the benthic habitat, the benthos was identified to functional group level rather than species. The mean values obtained for each functional group resident in each habitat at each station were used to create a dissimilarity matrix. A Bray-Curtis dissimilarity correlation was carried out on the data using Systat 10. The dissimilarity matrix was then used in a hierarchical cluster analysis, using the single linkage (nearest neighbour) method. The results of the cluster analysis were presented graphically and used to indicate stations with common characteristics. 


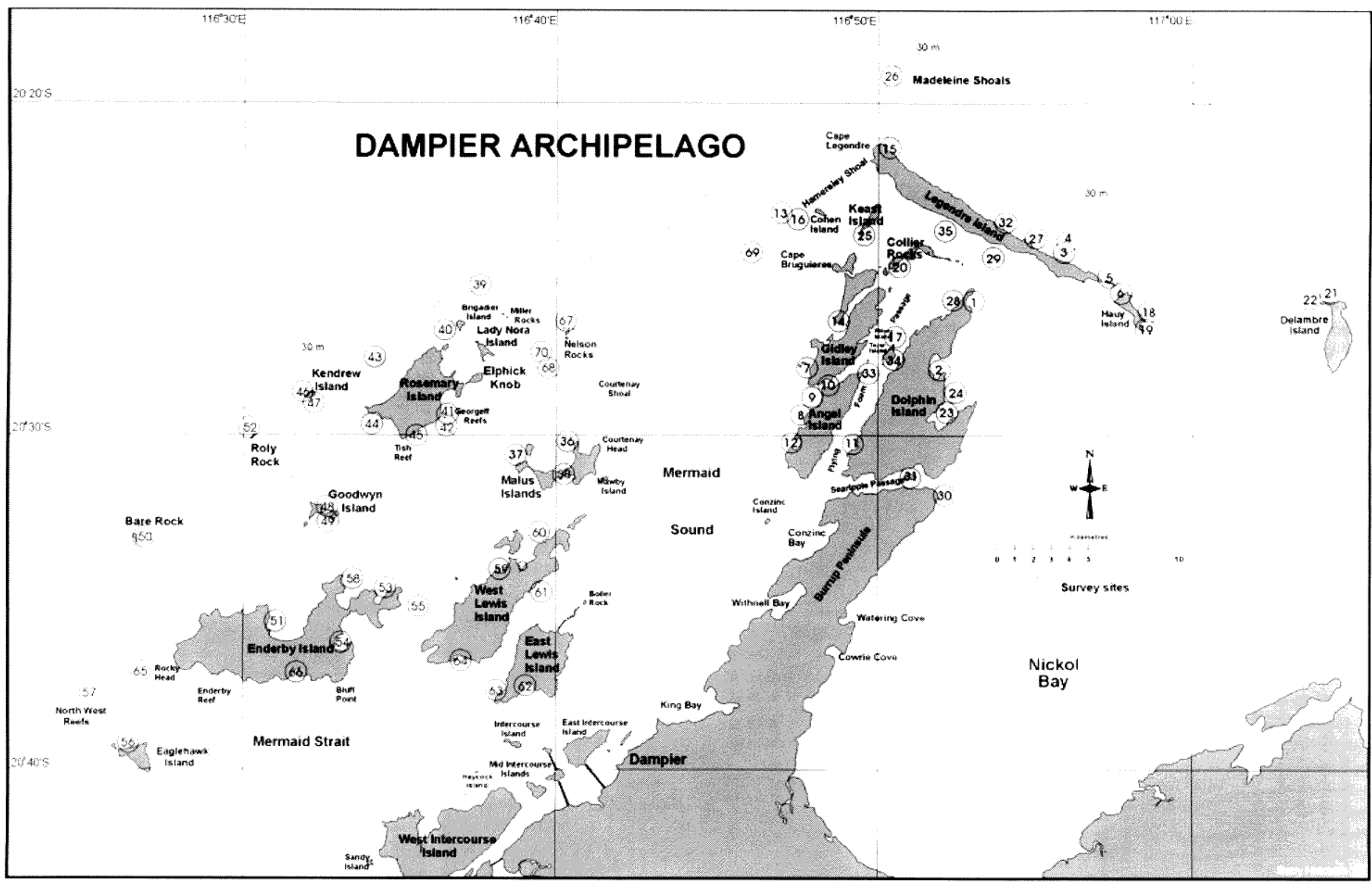




\section{STATION DESCRIPTIONS}

The raw percentage cover in terms of each benthic category by station is provided in Tables 3 and 4 . The analysis of percentage cover of benthic habitat was described in terms of the following categories:

1. Three abiotic categories (sand, rubble and rock).

2. One macroalgal category.

3. Three soft coral categories (alcyoniids, gorgonians and nephtheids).

4. Eight hard coral categories (branching Acropora, corymbose Acropora, digitate Acropora, tabulate Acropora, encrusting non-Acropora, foliaceous non-Acropora and massive non-Acropora).

5. Five additional categories (sponge, anemone, mollusc, echinoderm and ascidian).

6. One general category of 'other fauna'.

Of the above 20 categories, several were either poorly represented $(<0.5 \%$ cover $)$ or provided little information on their own. For this reason, in the presentation of the data in this report the three abiotic categories were combined and the five additional categories were added to the general category of 'other fauna'. The resulting presentation is, therefore, based on 13 categories of benthic habitat.

The habitat of each subtidal survey station is described in terms of percentage cover of each benthic category, as shown in Figures 2-4.

For the majority of the stations analysed, more than $50 \%$ of the surface area of the transect consisted of an abiotic benthic habitat (stations DA1/98/01, 03, 06, 08, 12, 13, 16, 18, 19, 21, 24, 26, $27,32,33,35$ and DA3/99/36, 37, 39, 41, 43, 44, 46, $47,49,50,53,55,56,57,58,63,64,65,67,68$ and 69) (Figure 2). The abiotic category is excluded in Figure 3 to better depict the living benthic habitat of the stations. In Figure 4, the three soft coral categories are combined into one and the eight hard coral categories are also combined into one.
The results of the hierarchical cluster analysis are shown in Figure 5. The analysis indicated several groupings of one or more stations. Stations DA1/ 98/03, 06, 16 and DA3/99/41, 43 and 68 were grouped based on their similarity in terms of an abiotic habitat, which was characteristic of these stations. Stations DA3/99/37, 47, 61 and 70 were grouped because they were dominated by macroalgae. Station DA3/99/65 was predominantly gorgonian soft coral while stations DA1/98/15 and 32, and DA3/99/39, 40 and 52 were grouped based on the percentage cover of neptheid and alcyoniid soft corals. Station DA3/99/ 60 was widely separated from the other stations, due to the large percentage cover by foliose nonAcropora hard corals. Stations DA1/98/22 and 29 formed a cluster based on their predominantly branching Acropora hard coral habitat while stations DA1/98/01 and 24, and DA3/99/50 and 63, were grouped due to the predominance of massive nonAcropora hard coral habitat.

The remaining 22 stations are difficult to separate further statistically without data from the various specimen collections. They were all dominated by abiotic and a combination of hard coral habitats, predominantly massive non-Acropora or tabulate Acropora species.

\section{REFERENCES}

Carleton, J.H. and Done, T.J. (1995). Quantitative video sampling of coral reef benthos: large scale application. Coral Reefs 14: 35-46.

Osborne, K. and Oxley, W.G. (1997). Sampling benthic communities using video transects. In English, S., Wilkinson, C. and Baker, V. (eds), Survey manual for tropical marine resources. The Australian Institute of Marine Science, Townsville, Queensland. 


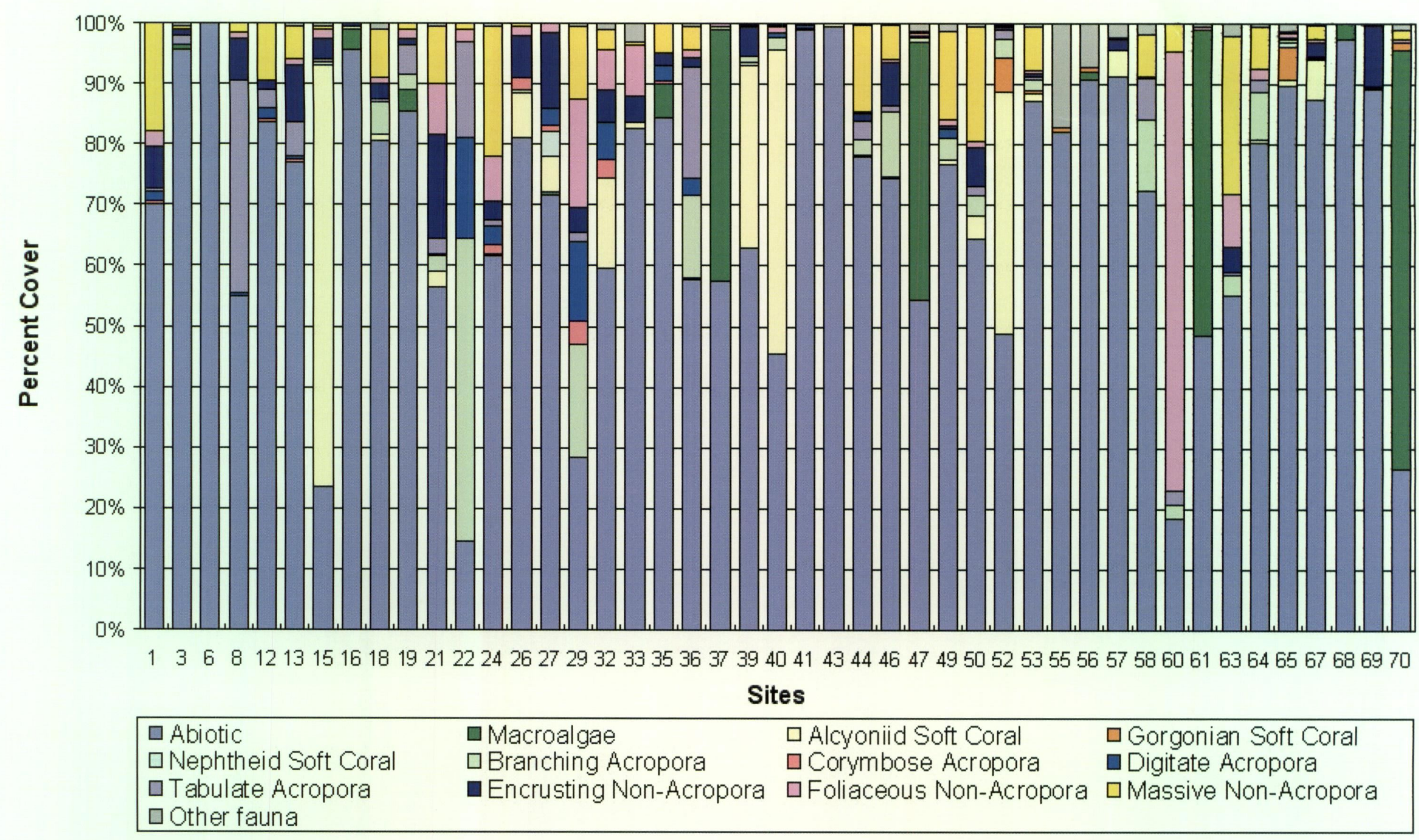

Figure 2 Percentage cover of habitat. 


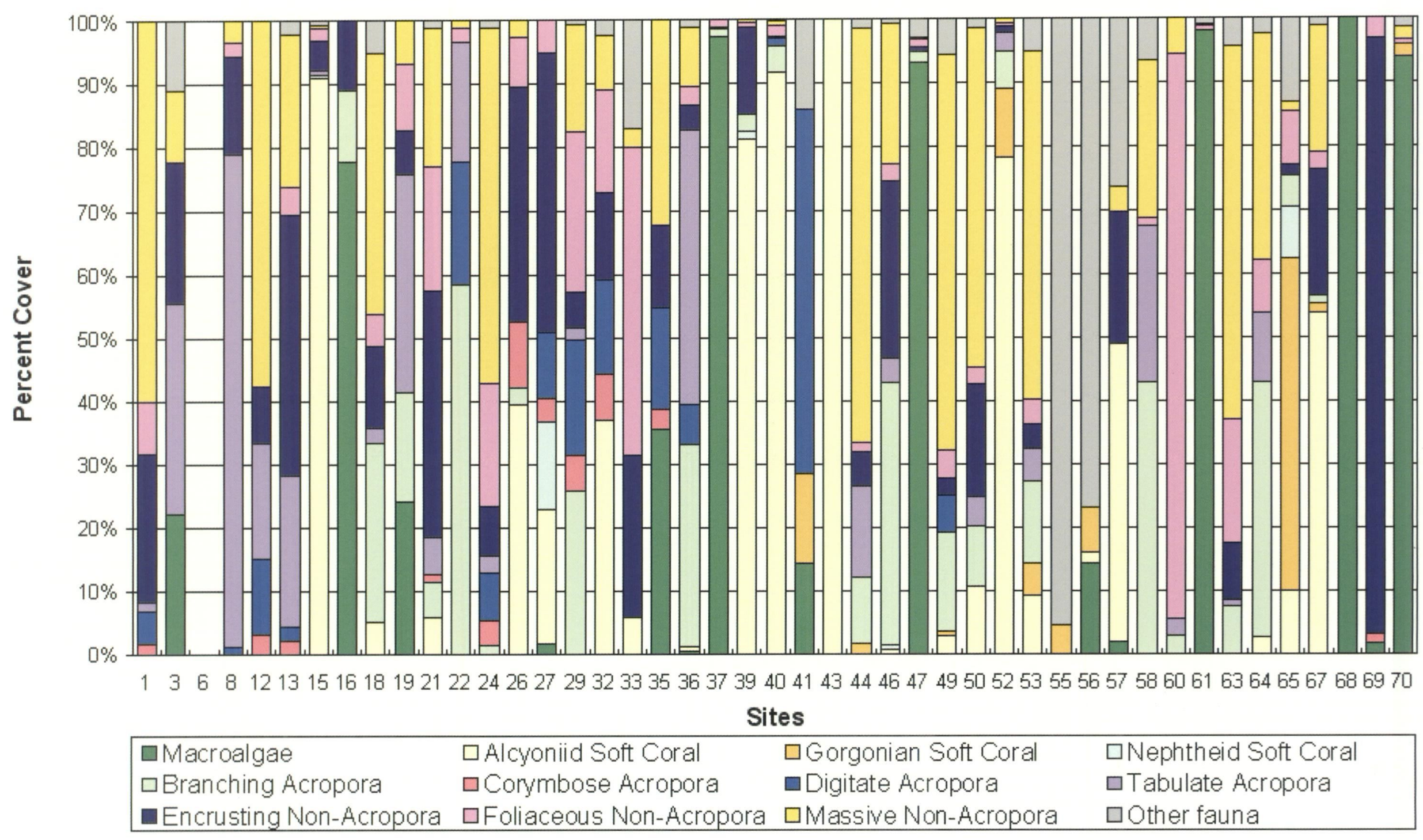




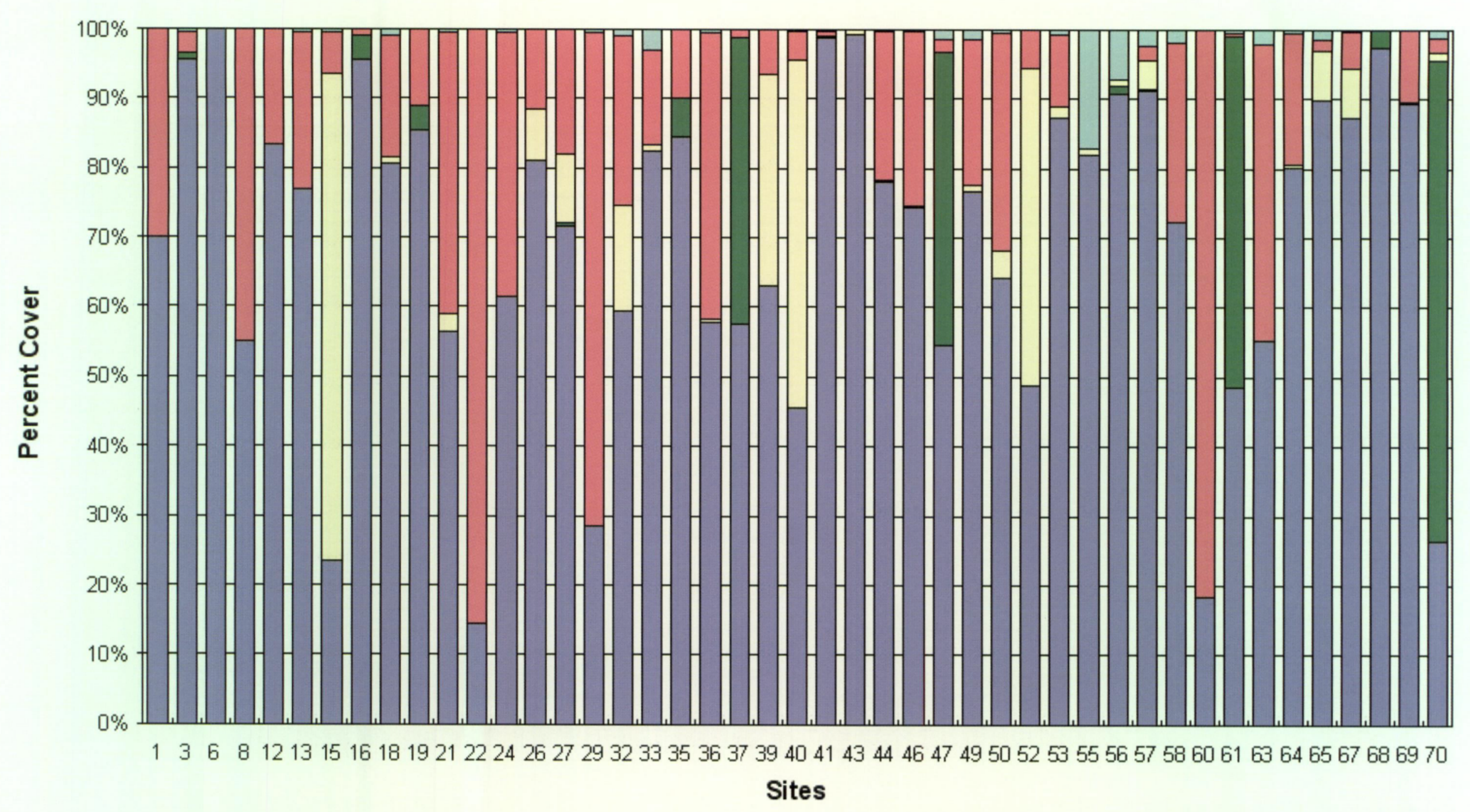

口Abiotic

-Macroalgae

$\square$ Soft Coral

a Hard Coral

口Other fauna

Figure 4 Percentage cover of combined habitat categories. 


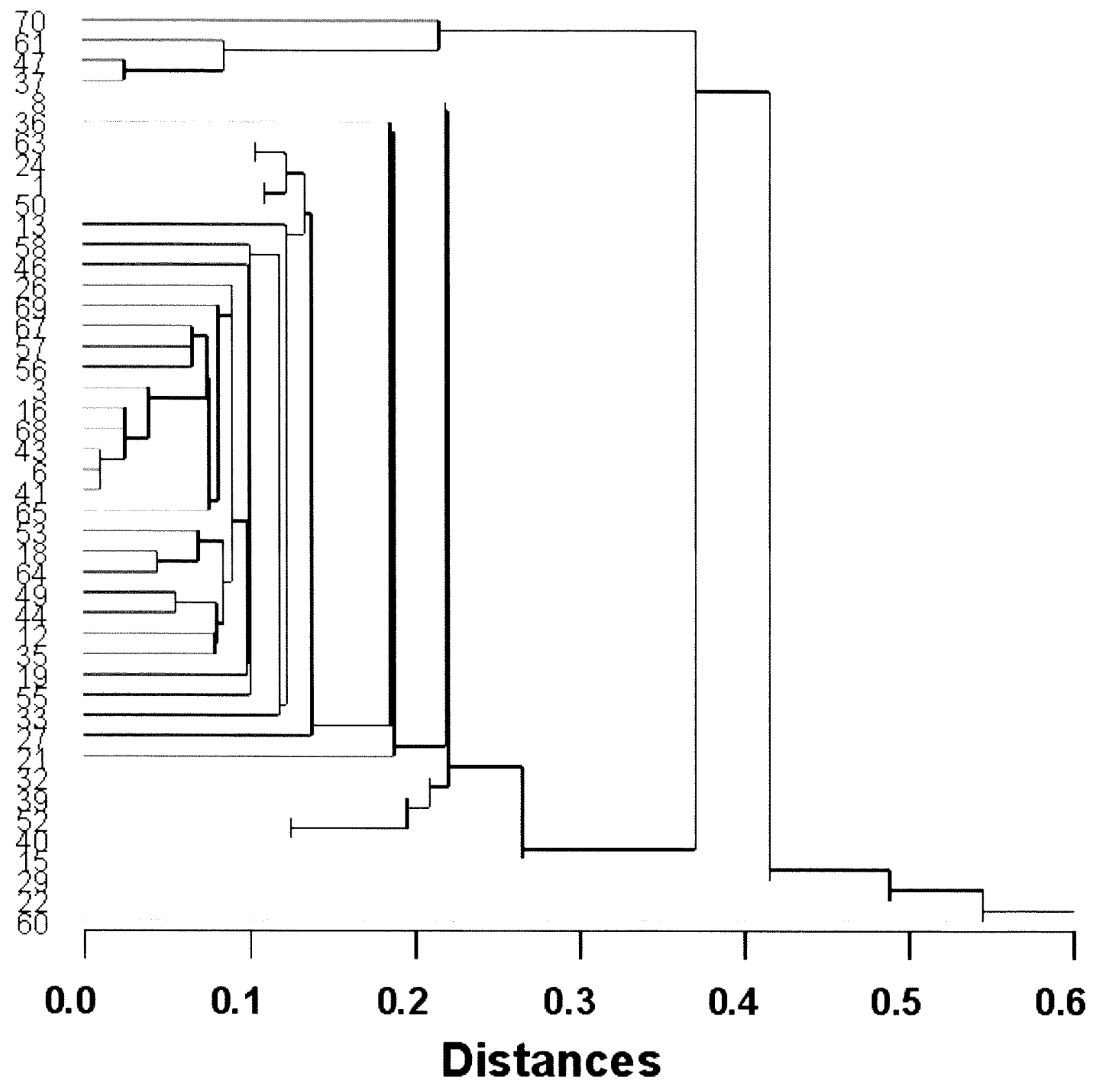

Figure 5 Cluster analysis. 
Table 1 Station descriptions for the DA1/98 diving survey.

\begin{tabular}{|c|c|c|c|c|c|}
\hline Station & Year & Habitat & Depth (m) & Latitude & Longitude \\
\hline DA1/98/01 & 1998 & Coral & 6 & $2025.852 ' S$ & $11652.953^{\prime} \mathrm{E}$ \\
\hline DA1/98/03 & 1998 & Coral on dissected limestone reef & 8 & $2024.320^{\prime} \mathrm{S}$ & $11656.108^{\prime} \mathrm{E}$ \\
\hline DA1/98/06 & 1998 & Patchy coral on limestone pavement & 3 & $2025.725 ' S$ & $11657.580^{\prime} \mathrm{E}$ \\
\hline DA1/98/08 & 1998 & Coral on dissected limestone reef & 4 & $2029.180^{\prime} S$ & $11647.711^{\prime} \mathrm{E}$ \\
\hline DA1/98/12 & 1998 & Patchy coral on limestone pavement & 6 & 2030.200 'S & $11647.249^{\prime} \mathrm{E}$ \\
\hline DA1/98/13 & 1998 & Coral on dissected limestone reef & 9 & 2023.203 'S & $11646.691 ' \mathrm{E}$ \\
\hline DA1/98/15 & 1998 & Soft coral on limestone reef & 16 & 2021.142 'S & $11650.579^{\prime} \mathrm{E}$ \\
\hline DA1/98/16 & 1998 & Coral regrowth on rubble & 4 & $2023.240^{\prime} \mathrm{S}$ & $11647.080^{\prime} \mathrm{E}$ \\
\hline DA1/98/18 & 1998 & Coral bommies on sand & 11 & 2026.400 'S & $11658.634^{\prime} \mathrm{E}$ \\
\hline DA1/98/19 & 1998 & Macroalgae and coral on sand and rubble & 3 & $2026.620^{\prime} \mathrm{S}$ & $11658.390^{\prime} \mathrm{E}$ \\
\hline DA1/98/21 & 1998 & Coral & 14 & $2025.700^{\prime} \mathrm{S}$ & $11704.220^{\prime} \mathrm{E}$ \\
\hline DA1/98/22 & 1998 & Coral & 5 & $2025.915 \mathrm{~S}$ & $11703.655^{\prime} \mathrm{E}$ \\
\hline DA1/98/24 & 1998 & Coral & 5 & 2028.870 'S & $11652.380^{\prime} \mathrm{E}$ \\
\hline $\mathrm{DA} 1 / 98 / 26$ & 1998 & Hard coral and soft coral on igneous rock & 22 & $2019.343^{\prime} S$ & $11650.455^{\prime} \mathrm{E}$ \\
\hline $\mathrm{DA} 1 / 98 / 27$ & 1998 & Hard coral and soft coral on limestone pavement & 16 & 2024.044 'S & $11655.041^{\prime} \mathrm{E}$ \\
\hline DA1/98/29 & 1998 & Coral & 4 & $2024.566^{\prime} \mathrm{S}$ & $11653.714^{\prime} \mathrm{E}$ \\
\hline DA1/98/32 & 1998 & Hard coral and soft coral on limestone reef & 14 & 2023.520 'S & $11654.110^{\prime} \mathrm{E}$ \\
\hline DA1/98/33 & 1998 & Soft coral and sponge on silt & 8 & 2027.965 'S & $11649.692^{\prime} \mathrm{E}$ \\
\hline DA1/98/35 & 1998 & Macroalgae and coral on sand & 1 & 2023.620 'S & $11651.960^{\prime} \mathrm{E}$ \\
\hline
\end{tabular}

Table 2 Station descriptions for the DA3/99 diving survey.

\begin{tabular}{|c|c|c|c|c|c|}
\hline Station & Year & Habitat & Depth (m) & Latitude & Longitude \\
\hline $\mathrm{DA} 3 / 99 / 36$ & 1999 & Coral & 9 & 2030.056 'S & $11640.579^{\prime} \mathrm{E}$ \\
\hline DA3/99/37 & 1999 & Macroalgae on rubble & 4 & $2030.612 ' S$ & $11638.918^{\prime} \mathrm{E}$ \\
\hline $\mathrm{DA} 3 / 99 / 39$ & 1999 & Soft coral on igneous rock & 16 & 2025.411 'S & $11637.613^{\prime} \mathrm{E}$ \\
\hline $\mathrm{DA} 3 / 99 / 40$ & 1999 & Soft corals & 8 & 2026.648 'S & $11636.522^{\prime} \mathrm{E}$ \\
\hline $\mathrm{DA} 3 / 99 / 41$ & 1999 & Coral bommies on sand & 4 & 2029.243 'S & $11636.974^{\prime} \mathrm{E}$ \\
\hline $\mathrm{DA} 3 / 99 / 43$ & 1999 & Patchy coral on limestone pavement & 11 & 2027.814 'S & $11634.192 ' \mathrm{E}$ \\
\hline $\mathrm{DA} 3 / 99 / 44$ & 1999 & Coral bommies on sand & 5 & 2029.590 'S & $11634.446^{\prime} \mathrm{E}$ \\
\hline $\mathrm{DA} 3 / 99 / 46$ & 1999 & Coral on dissected limestone reef & 5 & 2028.634 'S & $11631.991^{\prime} \mathrm{E}$ \\
\hline $\mathrm{DA} 3 / 99 / 47$ & 1999 & Macroalgae on limestone pavement & 4 & $2028.967 ' S$ & $11632.549^{\prime} \mathrm{E}$ \\
\hline $\mathrm{DA} 3 / 99 / 49$ & 1999 & Coral bommies on sand & 5 & 2032.433 'S & $11632.683^{\prime} \mathrm{E}$ \\
\hline $\mathrm{DA} 3 / 99 / 50$ & 1999 & Coral on dissected limestone reef & 15 & $2032.849^{\prime} \mathrm{S}$ & $11626.705^{\prime} \mathrm{E}$ \\
\hline $\mathrm{DA} 3 / 99 / 52$ & 1999 & Soft corals on limestone pavement & 15 & $2029.741^{\prime} \mathrm{S}$ & $11630.184^{\prime} \mathrm{E}$ \\
\hline $\mathrm{DA} 3 / 99 / 53$ & 1999 & Patchy coral on sand & 5 & $2034.528^{\prime} S$ & $11634.574^{\prime} \mathrm{E}$ \\
\hline $\mathrm{DA} 3 / 99 / 55$ & 1999 & Sponge and soft corals & 17 & 2035.111 'S & $11635.620^{\prime} \mathrm{E}$ \\
\hline $\mathrm{DA} 3 / 99 / 56$ & 1999 & Sponge and soft corals & 9 & $2038.939^{\prime} \mathrm{S}$ & $11626.218^{\prime} \mathrm{E}$ \\
\hline $\mathrm{DA} 3 / 99 / 57$ & 1999 & Soft coral on igneous rock & 9 & $2037.707 \mathrm{~S}$ & $11625.129^{\prime} \mathrm{E}$ \\
\hline $\mathrm{DA} 3 / 99 / 58$ & 1999 & Coral regrowth on rubble & 4 & $2034.386^{\prime} \mathrm{S}$ & $11633.558^{\prime} \mathrm{E}$ \\
\hline $\mathrm{DA} 3 / 99 / 60$ & 1999 & Coral & 3 & $2032.894 \mathrm{~S}$ & $11639.513^{\prime} \mathrm{E}$ \\
\hline $\mathrm{DA} 3 / 99 / 61$ & 1999 & Macroalgae and coral on sand and rubble & 5 & 2034.655 'S & $11639.721^{\prime} \mathrm{E}$ \\
\hline $\mathrm{DA} 3 / 99 / 63$ & 1999 & Coral & 2 & 2037.474 'S & $11638.288^{\prime} \mathrm{E}$ \\
\hline $\mathrm{DA} 3 / 99 / 64$ & 1999 & Patchy coral on sand & 5 & $2036.659^{\prime} \mathrm{S}$ & $11636.943^{\prime} \mathrm{E}$ \\
\hline $\mathrm{DA} 3 / 99 / 65$ & 1999 & Sponge and soft corals & 14 & 2037.096 'S & $11626.720^{\prime} \mathrm{E}$ \\
\hline $\mathrm{DA} 3 / 99 / 67$ & 1999 & Patchy coral on limestone pavement & 17 & $2026.505 ' S$ & $11640.225^{\prime} \mathrm{E}$ \\
\hline DA3/99/68 & 1999 & Sand with patchy soft coral and sponge & 7 & 2027.979 'S & $11639.733^{\prime} \mathrm{E}$ \\
\hline $\mathrm{DA} 3 / 99 / 69$ & 1999 & Rock armoured pipeline & 14 & $2024.466^{\prime} \mathrm{S}$ & $11646.298^{\prime} \mathrm{E}$ \\
\hline $\mathrm{DA} 3 / 99 / 70$ & 1999 & Macroalgae on pavement & 6 & $2027.448^{\prime} S$ & $11639.574^{\prime} \mathrm{E}$ \\
\hline
\end{tabular}


Table 3 Habitat percentage cover at each station during the DA1/98 diving survey.

\begin{tabular}{|c|c|c|c|c|c|c|c|c|c|c|c|c|c|c|c|c|c|c|c|}
\hline \multirow[t]{2}{*}{ Benthos type } & \multicolumn{19}{|c|}{ Stations } \\
\hline & 1 & 3 & 6 & 8 & 12 & 13 & 15 & 16 & 18 & 19 & 21 & 22 & 24 & 26 & 27 & 29 & 32 & 33 & 35 \\
\hline Abiotic & 70.0 & 95.5 & 100 & 55.0 & 83.5 & 77.0 & 23.5 & 95.5 & 80.5 & 85.5 & 56.5 & 14.5 & 61.5 & 81.0 & 71.5 & 28.5 & 59.5 & 82.5 & 84.5 \\
\hline Macroalgae & & 1.0 & & & & & & 3.5 & & 3.5 & & & & & 0.5 & & & & 5.5 \\
\hline Alcyoniid soft coral & & & & & & & 69.5 & & 1.0 & & 2.5 & & & 7.5 & 6.0 & & 15.0 & 1.0 & \\
\hline Gorgonian soft coral & & & & & & & & & & & & & & & & & & & \\
\hline Nephtheid soft coral & & & & & & & 0.5 & & & & & & & & 4.0 & & & & \\
\hline Branching Acropora & & & & & & & & 0.5 & 5.5 & 2.5 & 2.5 & 50.0 & 0.5 & 0.5 & & 18.5 & & & \\
\hline Corymbose Acropora & 0.5 & & & & 0.5 & 0.5 & & & & & 0.5 & & 1.5 & 2.0 & 1.0 & 4.0 & 3.0 & & 0.5 \\
\hline Digitate Acropora & 1.5 & & & 0.5 & 2.0 & 0.5 & & & & & & 16.5 & 3.0 & & 3.0 & 13.0 & 6.0 & & 2.5 \\
\hline Tabulate Acropora & 0.5 & 1.5 & & 35.0 & 3.0 & 5.5 & 0.5 & & 0.5 & 5.0 & 2.5 & 16.0 & 1.0 & & & 1.5 & & & \\
\hline Encrusting non-Acropora & 7.0 & 1.0 & & 7.0 & 1.5 & 9.5 & 3.5 & 0.5 & 2.5 & 1.0 & 17.0 & & 3.0 & 7.0 & 12.5 & 4.0 & 5.5 & 4.5 & 2.0 \\
\hline Foliaceous non-Acropora & 2.5 & & & 1.0 & & 1.0 & 1. & & 1.0 & 1.5 & 8.5 & 2.0 & 7.5 & 1.5 & 1.5 & 18.0 & 6.5 & 8.5 & \\
\hline Massive non-Acropora & 18.0 & 0.5 & & 1.5 & 9.5 & 5.5 & 0.5 & & 8.0 & 1.0 & 9.5 & 1.0 & 21.5 & 0.5 & & 12.0 & 3.5 & 0.5 & 5.0 \\
\hline Other fauna & & 0.5 & & & & 0.5 & 0.5 & & 1.0 & & 0.5 & & 0.5 & & & 0.5 & 1.0 & 3.0 & \\
\hline
\end{tabular}

Table 4 Habitat percentage cover at each station during the DA3/99 diving survey.

\begin{tabular}{|c|c|c|c|c|c|c|c|c|c|c|c|c|c|c|c|c|c|c|c|c|c|c|c|c|c|c|}
\hline \multirow[t]{2}{*}{ Benthos type } & \multirow[b]{2}{*}{36} & \multirow[b]{2}{*}{37} & \multirow[b]{2}{*}{39} & \multirow[b]{2}{*}{40} & \multirow[b]{2}{*}{41} & \multirow[b]{2}{*}{43} & \multirow[b]{2}{*}{44} & \multirow[b]{2}{*}{46} & \multirow[b]{2}{*}{47} & \multirow[b]{2}{*}{49} & \multicolumn{3}{|c|}{ Stations } & \multirow[b]{2}{*}{55} & \multirow[b]{2}{*}{56} & \multirow[b]{2}{*}{57} & \multirow[b]{2}{*}{58} & \multirow[b]{2}{*}{60} & \multirow[b]{2}{*}{61} & \multirow[b]{2}{*}{63} & \multirow[b]{2}{*}{64} & \multirow[b]{2}{*}{65} & \multirow[b]{2}{*}{67} & \multirow[b]{2}{*}{68} & \multirow[b]{2}{*}{69} & \multirow[b]{2}{*}{70} \\
\hline & & & & & & & & & & & 50 & 52 & 53 & & & & & & & & & & & & & \\
\hline Abiotic & 57.7 & 57.5 & 63.0 & 45.5 & 98.8 & 99.3 & 78.0 & 74.3 & 54.5 & 76.7 & 64.3 & 48.8 & 87.2 & 82.0 & 90.7 & 91.2 & 72.3 & 18.5 & 48.5 & 55.3 & 80.2 & 89.8 & 87.3 & 97.5 & 89.3 & 26.5 \\
\hline Macroalgae & 0.2 & +1.3 & 0.0 & 0.0 & 0.2 & 0.0 & 0.0 & 0.0 & 42.3 & 0.0 & 0.0 & 0.0 & 0.0 & 0.0 & 1.3 & 0.2 & 0.0 & 0.0 & 50.5 & 0.0 & 0.0 & 0.0 & 0.0 & 2.5 & 0.2 & 69.0 \\
\hline Alcyoniid soft coral & 0.3 & 0.0 & 30.0 & 50.0 & 0.0 & 0.7 & 0.0 & 0.2 & 0.0 & 0.7 & 3.8 & 40.0 & 1.2 & 0.0 & 0.2 & 4.2 & 0.0 & 0.0 & 0.0 & 0.0 & 0.5 & 1.0 & 6.8 & 0.0 & 0.0 & 0.0 \\
\hline Gorgonian soft coral & 0.0 & 0.0 & 0.0 & 0.0 & 0.2 & 0.0 & 0.3 & 0.0 & 0.0 & 0.2 & 0.0 & 5.5 & 0.7 & 0.8 & 0.7 & 0.0 & 0.0 & 0.0 & 0.0 & 0.0 & 0.0 & 5.3 & 0.2 & 0.0 & 0.0 & 1.3 \\
\hline Nephtheid soft coral & 0.0 & 0.0 & 0.5 & 0.0 & 0.0 & 0.0 & 0.0 & 0.2 & 0.0 & 0.0 & 0.0 & 0.0 & 0.0 & 0.0 & 0.0 & 0.0 & 0.0 & 0.0 & 0.0 & 0.0 & 0.0 & 0.8 & 0.0 & 0.0 & 0.0 & 0.0 \\
\hline Branching Acropora & 13.5 & 0.5 & 1.0 & 2.2 & 0.0 & 0.0 & 2.3 & 10.7 & 0.8 & 3.7 & 3.3 & 3.0 & 1.7 & 0.0 & 0.0 & 0.0 & 11.8 & 2.3 & 0.0 & 3.3 & 8.0 & 0.5 & 0.2 & 0.0 & 0.0 & 0.0 \\
\hline Corymbose Acropora & 0.0 & 0.0 & 0.0 & 0.0 & 0.0 & 0.0 & 0.0 & 0.0 & 0.0 & 0.0 & 0.0 & 0.0 & 0.0 & 0.0 & 0.0 & 0.0 & 0.0 & 0.0 & 0.0 & 0.0 & 0.0 & 0.0 & 0.0 & 0.0 & 0.2 & 0.0 \\
\hline Digitate Acropora & 2.7 & 0.2 & 0.0 & 0.7 & 0.7 & 0.0 & 0.0 & 0.0 & 0.0 & 1.3 & 0.0 & 0.0 & 0.0 & 0.0 & 0.0 & 0.0 & 0.0 & 0.0 & 0.0 & 0.0 & 0.0 & 0.0 & 0.0 & 0.0 & 0.0 & 0.0 \\
\hline Tabulate Acropora & 18.3 & 0.0 & 0.0 & 0.2 & 0.0 & 0.0 & 3.2 & 1.0 & 0.0 & 0.0 & 1.7 & 1.5 & 0.7 & 0.0 & 0.0 & 0.0 & 6.8 & 2.2 & 0.0 & 0.5 & 2.2 & 0.0 & 0.0 & 0.0 & 0.0 & 0.0 \\
\hline Encrusting non-Acropora & 1.7 & 0.0 & 5.0 & 0.0 & 0.0 & 0.0 & 1.2 & 7.2 & 0.3 & 0.7 & 6.3 & 0.5 & 0.5 & 0.0 & 0.0 & 1.8 & 0.0 & 0.0 & 0.0 & 4.0 & 0.0 & 0.2 & 2.5 & 0.0 & 10.0 & 0.0 \\
\hline Foliaceous non-Acropora & 1.2 & 0.5 & 0.3 & 1.0 & 0.0 & 0.0 & 0.3 & 0.7 & 0.5 & 1.0 & 1.0 & 0.3 & 0.5 & 0.0 & 0.0 & 0.0 & 0.3 & 72.3 & 0.3 & 8.7 & 1.7 & 0.8 & 0.3 & 0.0 & 0.3 & 0.7 \\
\hline Massive non-Acropora & 4.0 & 0.0 & 0.2 & 0.3 & 0.0 & 0.0 & 14.3 & 5.7 & 0.2 & 14.5 & 19.0 & 0.3 & 7.0 & 0.0 & 0.0 & 0.3 & 6.8 & 4.7 & 0.2 & 26.2 & 7.0 & 0.2 & 2.5 & 0.0 & 0.0 & 1.3 \\
\hline Other fauna & 0.5 & 0.0 & 0.0 & 0.2 & 0.2 & 0.0 & 0.3 & 0.2 & 1.3 & 1.3 & 0.5 & 0.0 & 0.7 & 17.2 & 7.2 & 2.3 & 1.8 & 0.0 & 0.5 & 2.0 & 0.5 & 1.3 & 0.2 & 0.0 & 0.0 & 1.2 \\
\hline
\end{tabular}

